\section{BRAZIULIAN JOURNAL}

OF MEDICAL AND BIOLOGICAL RESFARCH

www.bjournal.com.br
ISSN 0100-879X

Volume 43 (9) 812-913 September 2010

BIOMEDICAL SCIENCES

AND

CLINICAL INVESTIGATION

Braz J Med Biol Res, September 2010, Volume 43(9) 883-889

doi: 10.1590/S0100-879X2010007500077

\title{
A simple method to assess freezing of gait in Parkinson's disease patients
}

M.B. Popovic, M. Djuric-Jovicic, S. Radovanovic, I. Petrovic and V. Kostic

The Brazilian Journal of Medical and Biological Research is partially financed by
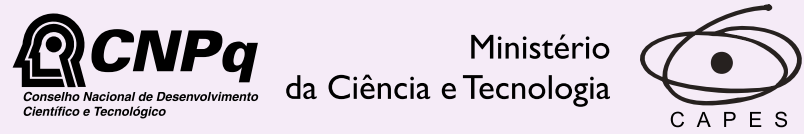

Ministério da Educação

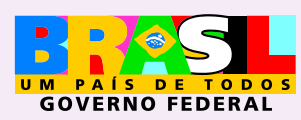

DFAPESP

Institutional Sponsors
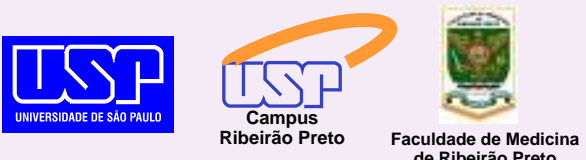

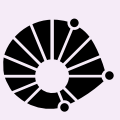

UNICAMP
Ф SHIMADZU

GE Healthcare
Hotsite of proteomics metabolomics developped by: 


\title{
A simple method to assess freezing of gait in Parkinson's disease patients
}

\author{
M.B. Popovic ${ }^{1,2,3}$, M. Djuric-Jovicic ${ }^{1,4}$, S. Radovanovic ${ }^{5}$, I. Petrovic ${ }^{6}$ and V. Kostic ${ }^{6}$ \\ ${ }^{1}$ University of Belgrade, School of Electrical Engineering, Belgrade, Serbia \\ 2Institute for Multidisciplinary Research, Belgrade, Serbia \\ ${ }^{3}$ Center for Sensory Motor Interaction, Department of Health Science and Technology, Aalborg University, Denmark \\ ${ }^{4}$ Fatronik Serbia, Belgrade, Serbia \\ ${ }^{5}$ Laboratory for Neurophysiology, Institute for Medical Research, Belgrade, Serbia \\ ${ }^{6}$ Institute of Neurology, University of Belgrade, School of Medicine, Clinical Centre of Serbia, Belgrade, Serbia
}

\begin{abstract}
Freezing of gait (FOG) can be assessed by clinical and instrumental methods. Clinical examination has the advantage of being available to most clinicians; however, it requires experience and may not reveal FOG even for cases confirmed by the medical history. Instrumental methods have an advantage in that they may be used for ambulatory monitoring. The aim of the present study was to describe and evaluate a new instrumental method based on a force sensitive resistor and Pearson's correlation coefficient (PcC) for the assessment of FOG. Nine patients with Parkinson's disease in the "on" state walked through a corridor, passed through a doorway and made a U-turn. We analyzed 24 FOG episodes by computing the Pcc between one "regular/ normal" step and the rest of the steps. The Pcc reached \pm 1 for "normal" locomotion, while correlation diminished due to the lack of periodicity during FOG episodes. Gait was assessed in parallel with video. FOG episodes determined from the video were all detected with the proposed method. The computed duration of the FOG episodes was compared with those estimated from the video. The method was sensitive to various types of freezing; although no differences due to different types of freezing were detected. The study showed that Pcc analysis permitted the computerized detection of FOG in a simple manner analogous to human visual judgment, and its automation may be useful in clinical practice to provide a record of the history of FOG.
\end{abstract}

Key words: Parkinson's disease; Freezing of gait; Time analysis; Pearson's correlation coefficient

\section{Introduction}

There are a growing number of papers concerning the freezing of gait (FOG) (1-7), which, however, is still a poorly understood phenomenon (8) commonly occurring among persons with advanced Parkinson's disease (PD). This characteristic extends beyond the classical manifestations of PD: akinesia, bradykinesia, rigidity, tremor, and postural instability. Freezing has been observed and quantified in internally cued repetitive movements such as gait, speech, handwriting, and manual tapping tasks as a distinct feature of PD.

FOG significantly disturbs mobility and deteriorates the functionality of gait. Freezing episodes in relation to gait occur most frequently when starting to walk, when turning, when approaching destinations or obstacles, in narrow spaces, and, less commonly, when walking in an open walkway $(1,3,9)$. FOG is not necessarily a completely frozen, akinetic posture. Thus, during locomotion, different characteristics of gait disturbances may be seen: feet may be "glued" to the ground (freezing) or they may change their normal rhythm (festination) (7,9-11). Much faster oscillations than the normal walking pattern may occur as the result of an ineffective effort to move forward. Gait festination is highly associated with FOG (10), suggesting that the two conditions may share a common pathophysiology such as a central timing mechanism (7). While glued to the ground, the lower extremities may show signs of shaking and asynchronous movement described as "trembling in place". Further studies are needed to determine the mechanisms responsible for the complex pattern during FOG and to investigate why FOG sometimes results in a frozen akinetic state and sometimes in complex movements.

Ambulatory monitoring may improve the clinical management of FOG (11-13). A reliable and easy method to study the frequency, timing, and intensity of FOG might

Correspondence: M.B. Popovic, University of Belgrade, School of Electrical Engineering, Bulevar kralja Aleksandra 73, 11000 Belgrade, Serbia. Fax: +381-11-3248-681. E-mail: mpo@etf.rs

Received October 26, 2009. Accepted August 3, 2010. Available online August 13, 2010. Published September 13, 2010. 
be used in clinical trials of new drugs or as a tool to study PD patients submitted to deep brain stimulation surgery with electrode implantation. A particularly difficult situation occurs when there is a lack of response to any medication used for PD treatment, especially in the beginning, when the response occurs only in the off periods or when this disturbance ceases to respond to levodopa. Monitoring gait may also possibly detect subclinical changes that could precede the FOG manifestation.

Gait with freezing episodes may be monitored by using different measurement systems: video $(5,11)$, insole forces (14), and accelerometers (11-13). The majority of gait analysis systems provide information about the force profile exerted by each foot on the ground during walking. In addition to time, frequency analysis was also evaluated $(4,11)$

Our choice to apply correlation analysis as a method to detect FOG was based on the theory considered by Rodgers and Nicewander (15) and Aldrich (16). Correlation is one of the oldest and most classical measures of interdependence between two time series $(17,18)$ and has already been used for various biosignals (19-21). The Pearson's correlation coefficient (Pcc) is a measure of the linear dependence between two signals. If the two signals have the same shape, the coefficient is 1 . By definition, the correlation between two periodic/quasiperiodic signals is high. On the contrary, if one signal is stochastic, the correlation diminishes due to the lack of periodicity. Any stochastic pattern of walking would diminish the Pcc value, and in our case it was FOG. For gait, given its quasiperiodic nature, the correlation between a "normal" step and the rest of the steps should decrease during FOG.

In the present paper, we propose a simple method for the detection of complex FOG episodes where Pcc analysis is applied to a series of stride force profiles recorded

Table 1. Patient demographics.

\begin{tabular}{lcccccc}
\hline Subject & Gender & Age (years) & $\begin{array}{c}\text { Time since } \\
\text { diagnosis (years) }\end{array}$ & H\&Y scale & \multicolumn{2}{c}{ Number of FOG episodes } \\
\cline { 5 - 7 } & & & & & 1st trial & 2nd trial \\
\hline 1 & $\mathrm{M}$ & 70 & 11 & 3.5 & 4 & 4 \\
2 & $\mathrm{M}$ & 57 & 4 & 4.0 & 6 & 4 \\
3 & $\mathrm{M}$ & 70 & 18 & 1.5 & 0 & 0 \\
4 & $\mathrm{M}$ & 77 & 14 & 2.0 & 1 & - \\
5 & $\mathrm{M}$ & 63 & 6 & 3.0 & 3 & - \\
6 & $\mathrm{M}$ & 79 & 4 & 3.0 & 1 & - \\
7 & $\mathrm{~F}$ & 78 & 14 & 2.5 & 1 & - \\
8 & $\mathrm{~F}$ & 63 & 15 & 1.5 & 0 & 0 \\
9 & $\mathrm{M}$ & 77 & 6 & 3.0 & 0 & 0 \\
Range & $7 \mathrm{M}+2 \mathrm{~F}$ & $57-79$ & $4-18$ & $1.5-4$ & $0-6$ & $0-4$ \\
Mean $\pm \mathrm{SD}$ & & $70.4 \pm 7.9$ & $10.2 \pm 5.3$ & $2.7 \pm 0.87$ & $1.8 \pm 1.84$ & $1.6 \pm 1.92$ \\
\hline
\end{tabular}

FOG = freezing of gait; $M=$ male; $F=$ female. $H \& Y=$ Hoehn and Yahr scale (Ref. 22). with force sensitive resistors (FSR). Consequently, FOG may be detected as times when the Pcc diminishes from values that are typical during normal locomotion. In parallel, we collected signals from accelerometers attached to the same leg and synchronized all signals with video. These additional recordings were used only for evaluation purposes (accelerometers) and validation (video) of the proposed method.

\section{Patients and Methods}

\section{Patients}

We analyzed data from 24 episodes of FOG in 9 patients with advanced PD. A clinical neurologist at the Institute of Neurology Clinical Centre of Serbia (INCCS) recruited 9 participants ( 7 males and 2 females). All patients reported a clinical history of FOG. Table 1 summarizes the demographic and clinical characteristics of the study population. The study was performed in accordance with the ethical standards of the Declaration of Helsinki. Institutional Ethics Committee approval was obtained and participants gave informed written consent prior to the inclusion in the experiment.

\section{Experimental protocol}

Patients arrived at noon at the INCCS, having taken their usual morning PD medications in an "on" state. One patient missed his morning dose. All but one of the participants walked without assistance at a self-paced speed along the corridor and a small room and then walked back the same way (Figure 1, left top). Patients were asked to stand up from the chair placed in the corridor (Figure 1, left bottom), walk toward the room, pass a doorway, turn $180^{\circ}$ to the left (U-turn) and walk the same route back, ending with a turn to sit back in the chair. The complex path included gait initiation, doorway passes, a U-turn, and a destination.

The distance walked was approximately 13 meters in each direction. Patients were asked to complete two trials separated by a rest period of at least $10 \mathrm{~min}$.

\section{Data acquisition}

During walking, movement of the more affected leg was evaluated using FSR (23) and a custom-made acquisition system using accelerometers $(24,25)$.

Four FSR sensors were mounted on the shoe insole in a standard manner (Figure 1, right top). In parallel, six 3-axis ADXL330 (Analog Devices, USA) accelerometers 
with appropriate signal conditioning circuitry were secured to the ends of 3 light bars (26). The bars were attached with tape along the foot, the shank and thigh (Figure 1, right top and bottom). The accelerometers were positioned so that one direction of the sensor pointed along the length of the bar, while the other two directions pointed perpendicular to it. The data used in this study were collected from the measurements in the direction perpendicular to the axes of foot, shank and thigh only. The leads from the six accelerometers were fastened to the foot, shank and thigh, leaving enough slack so as not to hinder motion.

The voltages from the FSR and the accelerometers were recorded by a portable custom-made data acquisition system consisting of a battery-operated mobile unit and a stationary data-acquisition computer at a rate of 166 samples per second, with a 10-bit resolution (27). The wireless link between the mobile and stationary unit was based on the Bluetooth standard. The maximal range of the link was $100 \mathrm{~m}$. The stationary unit was a remote PCbased computer with the appropriate software to control the measurement and acquisition procedure (start, stop, and the channels to be monitored). The software could be used for online monitoring of the measurement signals as well as for the recording of acquired signals and displaying saved signals (27). The mobile unit was a small, light device that communicated with the stationary unit and was worn by the subject while walking (Figure 1, bottom left).

All walking trials were recorded with a digital video camera (Canon, G7). Synchronization of data from the acquisition system with the video recordings was achieved by obtaining an image frame of the LED that lit up when the data logging was initiated (Figure 1, left bottom).

\section{Data analysis}

Periods of FOG episodes were studied from the three sources: video recordings, ground reaction forces and accelerations. Raw data were continuously visually inspected and processed with MatLab (ver. 7.3.0 (2006b), MathWorks Inc., USA). Signals were low-pass filtered with a 4th-order Butterworth filter using a cut-off frequency of $10 \mathrm{~Hz}$.

We analyzed the time series of ground reaction forces with Pcc (FSR). In the analyzing procedure, one step from the sequence of "normal" locomotion was first selected. This step was used to compute the correlation coefficient by means of Pcc with the entire gait record. Steps from the FSR records yielding Pearson's peaks that were deviated from \pm 1 were considered to be "irregular". We hypothesized that the decrease of these peaks was the result of complex freezing episodes.

\section{Evaluation/validation}

Video recordings were used for method validation. The computed Pcc was plotted in parallel to the video signal and FSR record. Visually identified FOGs from the video were compared with those determined via Pcc (FSR). In this study, accelerations were only used for the confirmation of the gait pattern. Finally, we compared the duration of FOG intervals determined by the two methods, Pcc (FSR) and video. The relative difference in duration was estimated with the following equation: $\Delta=\left(t_{\text {video }}-t_{p c c}\right) / t_{\text {video }}$, and reported as percent.

\section{Results}

A total of 24 FOG episodes occurred during 14 trials of 9 subjects (Table 1). Patients experienced episodes of FOG ranging from 0 to 6 per subject, per trial (Table 1, last two columns). Three patients (\#3, \#8, and \#9) did not freeze during the experiment. Four patients (\#4-\#7) completed one trial only. We applied the Pcc method to all FSR records.

An example of FOG detection with different sensors is illustrated in Figure 2. Video (a), accelerations from the foot, shank and thigh (b) and ground reaction forces from the foot (c) all revealed a FOG episode. When Pcc was
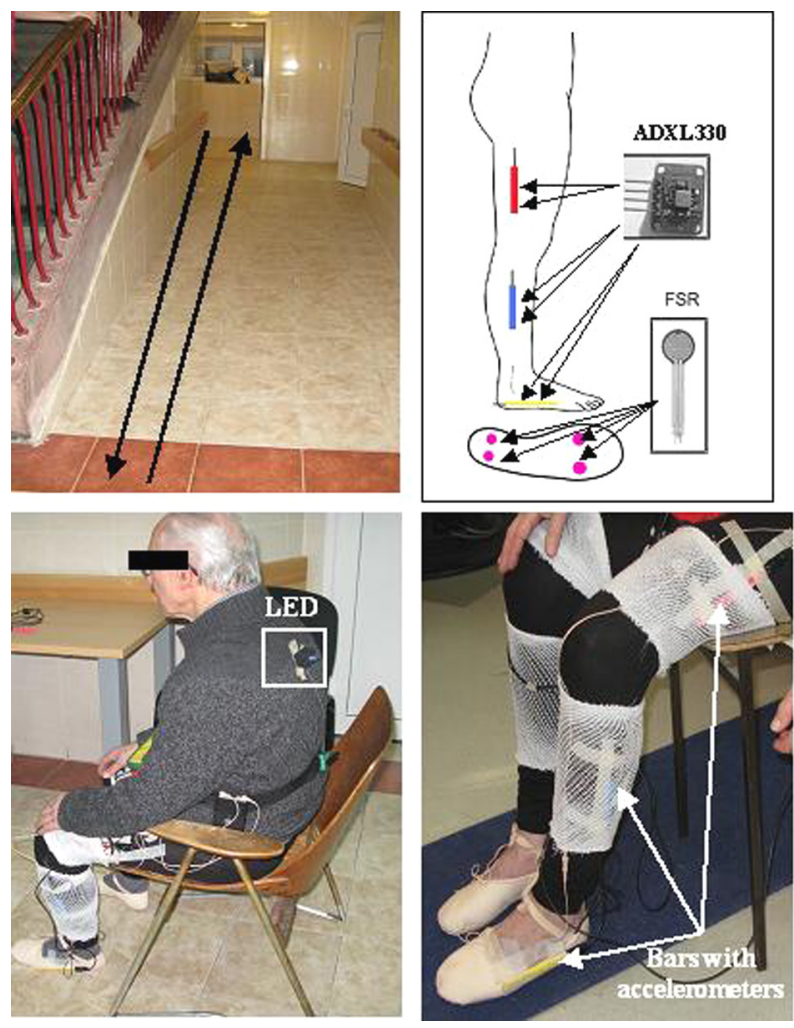

Figure 1. Experimental setup. Left top, Patients walked through the corridor, passed through a doorway, made a $180^{\circ}$ left turn, and walked back the same way. Right top, Sensors used: ADXL330 and FSR (23-25). Left bottom, Starting position. The LED for synchronization between the acquisition system (19) and the video is in the white rectangle. Right bottom, Red, blue, and yellow bars with pairs of accelerometers are attached to the thigh, shank, and foot (26). FSR = force sensitive resistor. 


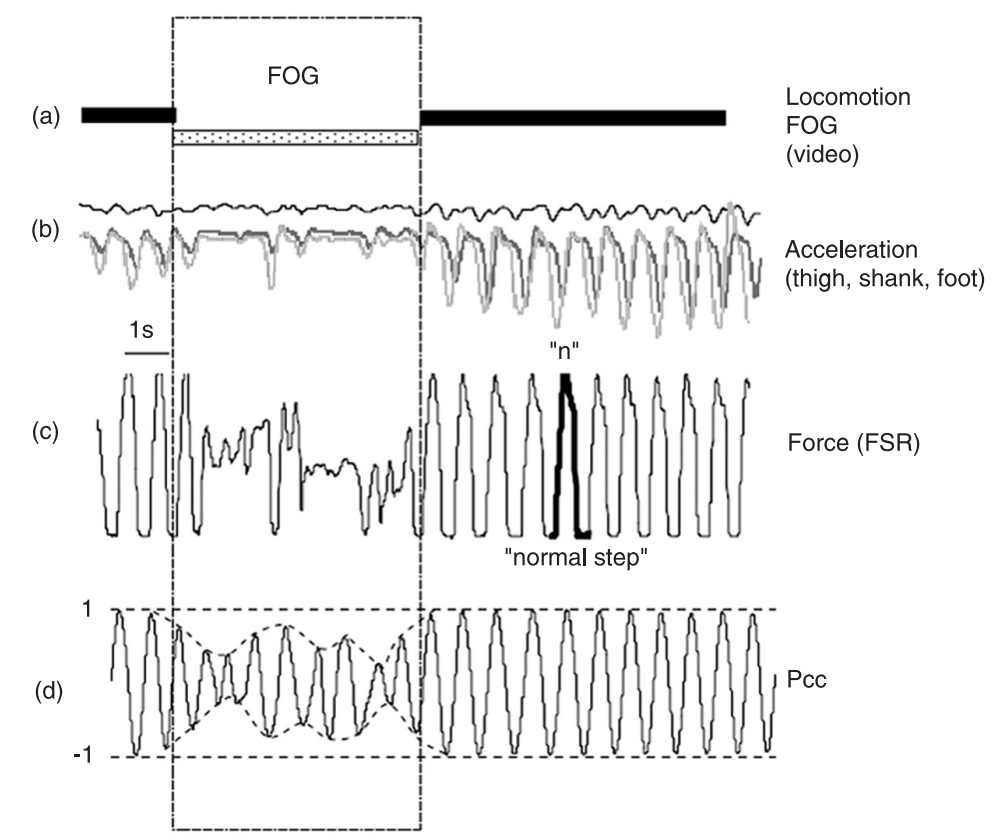

Figure 2. An example of freezing of gait (FOG) detection using Pearson's correlation coefficient (Pcc) analysis. a, Locomotion (dark bar) and FOG (light bar) episode determined from the video for validation of the method. $b$, Acceleration of the right thigh (black line), shank (dark gray line), and foot (light gray line) is quiet during FOG. $c$, The signal of the ground reaction force was computed as the sum of signals from 4 FSR sensors attached to the right shoe insole. One "regular/normal" step (thick line $\mathrm{n}$ ) was selected. $d$, Pcc was computed between a "normal/regular" step (thick line $n$ ) and the full record from $c$. Extreme Pcc values oscillate between -1 and 1 . A linear envelope (dashed line) was created from Pcc peaks with values under \pm 1 . Note: The large window (dotted rectangle) indicates FOG in $a, b, c$, and $d$. Data are for Parkinson's disease subject \#2 in a second trial. FSR $=$ force sensitive resistor.

(a)

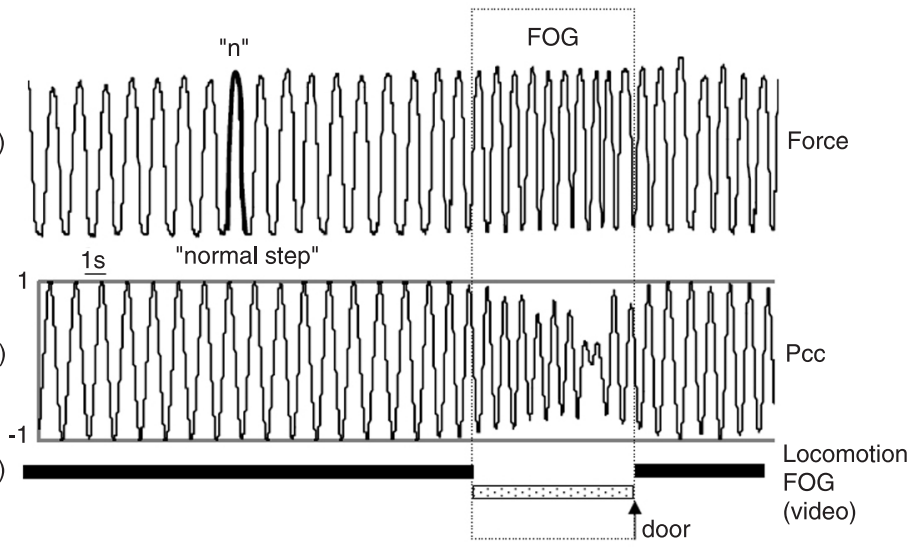

Figure 3. Example of the application of Pearson's correlation coefficient (Pcc) to walking with characteristic "small steps". a, Force from insole and a selected "normal" step (thick line n). $b$, Pcc computed between a "normal" step and the entire record oscillates around \pm 1 except during "small steps". c, The video shows locomotion (dark bars) and an episode with "small steps" (light bar) during which the Pcc range was reduced. Note: The large window (dotted rectangle) depicts a FOG episode in $a, b$, and $c$. Data are for Parkinson's disease patient \#5 in the first trial while the subject was approaching the door. FOG = freezing of gait. applied to the FSR (d), a decrease of Pcc peaks was noticeable during the FOG episode. Agood match between $a, b, c$, and $d$ is emphasized in the dashed window. The data illustrated in Figure 2 were collected from PD patient \#2 in a second trial.

An example of the application of Pcc to normal locomotion with a period of "small steps" is illustrated in Figure 3. First, one "normal" step (thick line) was selected from the force record during regular locomotion (a). Subsequently, Pearson's correlation coefficient was computed between the selected normal step and the entire record (b). Pcc values reached \pm 1 , except during "small steps". This irregularity occurred while the patient approached the door and was confirmed with the video (Figure $3 \mathrm{c}$, light bar at bottom). The period of FOG with "small steps" is highlighted in the dotted window in Figure 3. The relative difference between the times of the FOG episode, as determined by the two methods, video and Pcc, was less than $0.7 \%$. The data illustrated in Figure 3 were collected from PD patient \#5 in the first trial.

An example of the Pcc-based evaluation of freezing episodes in relation to several different obstacles is presented in Figure 4. This procedure involved the selection of a "normal/ regular" step (thick line) from a plot of ground reaction forces (a) followed by the computation of the Pcc (b). During normal walking, Pcc values oscillated between \pm 1 , and just before the obstacles \pm 1 peaks were observed. This result occurred six times during this record, as shown in Figure 4. These times were compared to the times of FOG episodes estimated from the video (Figure 4c, light bars at bottom). The relative difference for 6 FOG episodes was, at most, $0.6 \%$. The data presented in Figure 4 were collected from PD patient \#2 in the first trial. Figure 4 also illustrates various irregular freezing patterns observed. Akinetic freezing was observed before Door 1; "small steps" were observed in four instances (ahead of Line 1, Door 2, Line 2 and approaching the Chair); and asynchronous complex movement, followed by "small steps", was observed during turning. The gait pattern was also slightly altered while patients approached an array of red floor tiles (Figure 1, left top). This was defined as line 1 in one walking direction and line 2 in the opposite direction.

Steps used in the computation of the Pcc were visually selected from periods of "normal" locomotion. Figure 5 illustrates the effect of this 
selection. For the illustration, two steps - "normal" (thick line n) and "freezing" (thick line f) - were selected (a), and "Pcc n" (b) and "Pccf" (c) were computed, respectively. A video was recorded in parallel (d). The difference between peaks of "Pcc n" and "Pcc f" is noticeable. In "Pcc n", but not in "Pcc $f$ ", peaks were within the \pm 1 interval during normal walking. The FOG interval was noticeable for "Pcc n". When compared to the video record, the relative difference in duration was less than $0.4 \%$ maximum.

We compared the Pcc (FSR) method with the video. FOG episodes identified from the video corresponded to those detected with Pcc (FSR). The times of all 24 FOG episodes were evaluated using Pcc and compared to the times estimated from the video, as shown in Table 2. The relative mean $( \pm S D)$ difference in percentage between the two methods was $0.84 \pm 0.39$. The signals from the accelerometers followed these results and are included in a separate publication. Finally, the method was sensitive to the value of Pcc (Table 3) that was used for the determination of FOG duration. These rather small differences may have been patient specific.

\section{Discussion}

Nine PD patients with a medical history of FOG wore force-sensitive insoles that assessed gait during comfortable walking. In parallel, gait was assessed with accelerometers fixed to bars attached to the patients' leg segments. Each session was synchronized with video. Three sources (video, accelerometer (ACC), FSR sensors) were used in this study in order to obtain parallel records of FOG. The objective was to use one sensor (FSR or ACC) with a simple mathematical algorithm and to validate these findings using the video. Here, we proposed a simple mathematical algorithm (Pearson's correlation coefficient) (a)
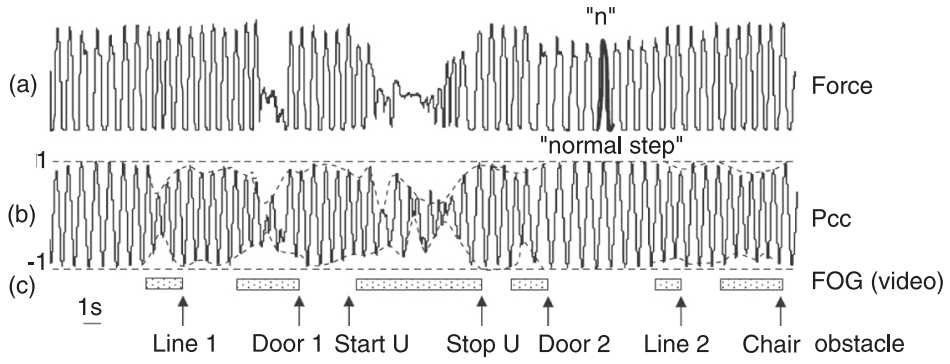

Figure 4. Illustration of the Pearson's correlation coefficient (Pcc) method in different walking situations. $a$, "Normal/regular" step was selected (thick line n) from a ground reaction force; $b$, Pcc was computed from $a$. Peaks oscillate around \pm 1 during locomotion and decrease just ahead of various obstacles. $c$, Six FOG episodes from the video (light bars) during which the Pcc range was reduced. Note: Line, Door, U-turn (Start-Stop), and Chair are indicated with arrows at the bottom. Index 1 (Door 1, Line 1) is for one walking direction and index 2 (Door 2, Line 2) is for the opposite direction (Figure 1, left top). Data are for Parkinson's disease patient \#2 in the first trial. FOG = freezing of gait.

(a)
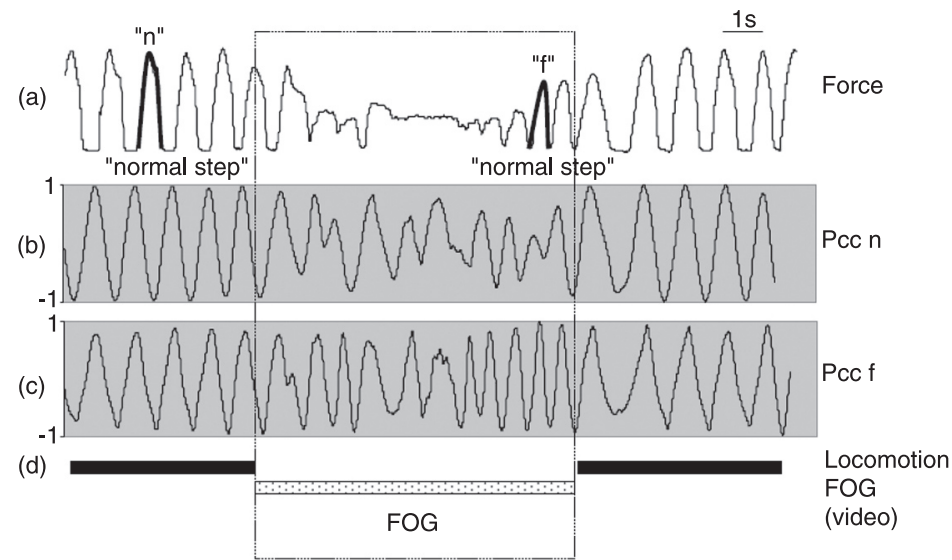

Figure 5. Selection of a step for the computation of Pearson's correlation coefficient (Pcc). a, "Normal" (thick line $n$ ) and "freezing" (thick line f) steps selected from walking and freezing episodes, respectively; $b$, "Pcc n" computed from a with a "normal" step; $c$, "Pcc f" computed from a with a "freezing" step; $d$, Locomotion (dark bar) and freezing (light bar) were estimated from the video. The reduction of the Pcc range matched freezing in $b$, but not in $c$. Please note the difference between peaks of "Pcc $n$ " and "Pcc $f$ ". FOG $=$ freezing of gait.
Table 2. Comparison of Pcc (FSR) with video data.

\begin{tabular}{lccc}
\hline $\begin{array}{l}\text { Duration of } \\
\text { FOG episode }\end{array}$ & Video [s] & $\begin{array}{c}\text { FSR } \\
(\mathrm{Pcc} \leq 0.9)[\mathrm{s}]\end{array}$ & $\begin{array}{c}\text { Relative } \\
\text { difference } \Delta[\%]\end{array}$ \\
\hline $\mathrm{T}_{\mathrm{FOG} 1}$ & 3.81 & 3.85 & 1.05 \\
$\mathrm{~T}_{\mathrm{FOG} 2}$ & 1.86 & 1.87 & 0.54 \\
$\quad \vdots$ & & & \\
$\mathrm{T}_{\mathrm{FOG} 24}$ & 9.33 & 9.23 & 1.07 \\
Mean $\pm \mathrm{SD}$ & $5.43 \pm 1.69$ & $5.60 \pm 1.68$ & $0.84 \pm 0.39$ \\
\hline
\end{tabular}

$\mathrm{Pcc}=$ Pearson's correlation coefficient; FSR = force sensitive resistor; FOG = freezing of gait.
Table 3. Sensitivity analysis.

\begin{tabular}{lc}
\hline $\begin{array}{l}\text { Pearson's correlation } \\
\text { coefficient, Pcc }\end{array}$ & $\begin{array}{c}\text { Relative difference } \Delta[\%] \\
\text { (mean } \pm \text { SD) }\end{array}$ \\
\hline 0.85 & $0.89 \pm 1.02$ \\
0.90 & $0.84 \pm 0.39$ \\
0.95 & $1.70 \pm 1.45$ \\
\hline
\end{tabular}


employing FSR sensors only. With FSR sensors built into the shoe insoles, gait was recordable at any time of walking. FOG was remotely recognizable on-line through the use of Bluetooth technology. As expected, the results showed that PD patients displayed an abnormal walking pattern of an episodic nature. This characteristic makes gait a complex motor task to analyze.

This study demonstrated the use of a simple method for the detection of complex FOG episodes from normal locomotion by computing Pearson's correlation coefficient between one "regular" step and the entire record of ground reaction forces from the patient's foot. This easy mathematical tool provided information about walking unsteadiness. It was sensitive to various freezing patterns such as sudden akinetic posture with or without asynchronous leg movement, as well as to "small steps" or "shuffling". On this basis, it did not distinguish between these two motions. Further development of this spatial-temporal measure is needed. Specifically, the method may be further automated to create information about FOG incidences instantly, but numerical parameters for the decision rules need to be analyzed and specified in a larger study.

Our setup included various gait obstacles. For example, because FOG most often occurs during turns and at gait initiations, these were included in the study. Other situations should also be evaluated. Two patients changed gait rhythm ("small steps") while crossing a line on the ground - kinesia paradoxa (28). This incidence should also be studied further.

Our opinion is that this simple, but reliable method may

\section{References}

1. Giladi N, McMahon D, Przedborski S, Flaster E, Guillory S, Kostic V, et al. Motor blocks in Parkinson's disease. Neurology 1992; 42: 333-339.

2. Giladi N, McDermott MP, Fahn S, Przedborski S, Jankovic $\mathrm{J}$, Stern M, et al. Freezing of gait in PD: prospective assessment in the DATATOP cohort. Neurology 2001; 56: 17121721.

3. Giladi N, Treves TA, Simon ES, Shabtai H, Orlov Y, Kandinov $B$, et al. Freezing of gait in patients with advanced Parkinson's disease. J Neural Transm 2001; 108: 53-61.

4. Hausdorff JM, Balash Y, Giladi N. Time series analysis of leg movements during freezing of gait in Parkinson's disease: rhyme or reason? Physica A 2003; 321: 565-570.

5. Hausdorff JM, Schaafsma JD, Balash Y, Bartels AL, Gurevich T, Giladi N. Impaired regulation of stride variability in Parkinson's disease subjects with freezing of gait. Exp Brain Res 2003; 149: 187-194.

6. Schaafsma JD, Balash Y, Gurevich T, Bartels AL, Hausdorff JM, Giladi N. Characterization of freezing of gait subtypes and the response of each to levodopa in Parkinson's disease. Eur J Neurol 2003; 10: 391-398.

7. Okuma Y. Freezing of gait in Parkinson's disease. J Neurol 2006; 253 (Suppl 7): VII27-VII32. provide easy access to objective results in the analysis of a particular treatment directed at this phenomenon. In the long run, this may eventually provide further insight into this specific disturbance of motor programming.

Thus, we foresee a great utility for the method described here. The method may be used to complement clinical examination, and we suggest that this may involve better reliability, greater efficiency and easy access to the results of assessment.

The use of a simple wearable system (FSR) and a reliable, yet fast algorithm (Pcc) in a wireless environment (in a clinical setting or outside) offers an easy tool for continuous follow-up of a specific medical treatment of FOG. We believe that if a standardized series of walking tasks can be recorded equally in different patients, thereby permitting a comparison between these patients before and after a therapeutic intervention, then a reliable method would emerge for the objective study of their gait pattern.

Finally, in this study we were able to demonstrate that Pcc applied to a force-time series recorded from the foot was capable of isolating FOG episodes from normal locomotion. Our results showed the feasibility of using Pcc in the ambulatory setting.

\section{Acknowledgments}

Research supported by the Ministry of Science, Serbia (\#175016, \#156011 and \#145057); the Danish National Research Foundation, Denmark, and the authors' respective institutions.

8. Jankovic J. Parkinson's disease: clinical features and diagnosis. J Neurol Neurosurg Psychiatry 2008; 79: 368-376.

9. Bartels AL, Balash Y, Gurevich T, Schaafsma JD, Hausdorff JM, Giladi N. Relationship between freezing of gait (FOG) and other features of Parkinson's: FOG is not correlated with bradykinesia. J Clin Neurosci 2003; 10: 584-588.

10. lansek R, Huxham F, McGinley J. The sequence effect and gait festination in Parkinson disease: contributors to freezing of gait? Mov Disord 2006; 21: 1419-1424.

11. Moore ST, MacDougall HG, Ondo WG. Ambulatory monitoring of freezing of gait in Parkinson's disease. $J$ Neurosci Methods 2008; 167: 340-348.

12. Moore ST, MacDougall HG, Gracies JM, Cohen HS, Ondo WG. Long-term monitoring of gait in Parkinson's disease. Gait Posture 2007; 26: 200-207.

13. Weiss A, Inbar-Borovsky N, Maidan I, Brozgol M, Plotnik M, Giladi N, et al. Can an accelerometer enhance Timed-Up \& Go test sensitivity among patients with Parkinson's disease? Mov Disord 2009; 24 (S1): 389.

14. Hausdorff JM, Cudkowicz ME, Firtion R, Wei JY, Goldberger AL. Gait variability and basal ganglia disorders: stride-tostride variations of gait cycle timing in Parkinson's disease and Huntington's disease. Mov Disord 1998; 13: 428-437. 
15. Rodgers JL, Nicewander WA. Thirteen ways to look at the correlation coefficient. Am Stat 1988; 42: 59-66.

16. Aldrich J. Correlations genuine and spurious in Pearson and Yule. Stat Sci 1995; 10: 364-376.

17. Bruce EN. Biomedical signal processing and signal modeling. New York: John Wiley and Sons; 2001.

18. Naït-Ali A. Advanced biosignal processing. Berlin; Heidelberg: Springer; 2009.

19. Xu W, Chang C, Hung YS, Kwan SK, Chin Wan Fung P. Order statistics correlation coefficient as a novel association measurement with applications to biosignal analysis. IEEE Trans Signal Process 2007; 55: 5552-5563.

20. Saul P. Beat-to-beat variations of heart rate reflect modulation of cardiac autonomic outflow. News Physiol Sci 1995; 5: 32-37.

21. Pereda E, Quian Quiroga R, Bhattacharya J. Nonlinear multivariate analysis of neurophysiological signals. Prog Neurobiol 2005; 77: 1-37.

22. Hoehn MM, Yahr MD. Parkinsonism: onset, progression and mortality. Neurology 1967; 17: 427-442.

23. Bijelic G, Dinic D, Popovic MB. Novel system for detection and acquisition of gait parameters. Proceedings of 50th Conference for Electronics, Telecommunication, Computers,
Automation and Nuclear Physics ETRAN. Vol III, ME 1.3. Serbia: 2006. p 227-230.

24. Mijovic B, Popovic MB, Popovic DB. Synergistic control of forearm based on accelerometer data and artificial neural networks. Braz J Med Biol Res 2008; 41: 389-397.

25. Mitrovic U, Malesevic N, Milovanovic I, Popovic MB. Evaluation of the system based on ADXL311 accelerometer to detect the swing phase in walking. Proceedings of the 51st Conference for Electronics, Telecommunication, Computers, Automation and Nuclear Physics ETRAN.ME1.7, [CD-ROM 4 p.], ISBN 978-86-80509-62-4 (granted the Best Young Researcher's Award in Biomedicine). Herceg Novi - Igalo, Monte Negro: 2007.

26. Iftime Nielsen SD, Popovic MB, Popovic DB. Estimation of joint angles with capacitive accelerometer. Proceedings of the Annual IEEE Student Paper Conference. [CD-ROM: 4p.], ISBN Print: 978-1-4244-2156-5. Aalborg, Denmark; 2008.

27. Jovicic N, Djuric M, Popovic DB. Portable data acquisition system for gait analysis based on Bluetooth communication. Proceedings of the 15th Conference for Telecommunication Forum TELFOR. Belgrade, Serbia: 2007. p 484-487.

28. Martin JP. The basal ganglia and posture. London: Pitman Medical Publishers; 1967. 